\title{
MUJERES EN ESPACIOS DE DECISIÓN. ESCENARIO ACTUAL EN LAS UNIVERSIDADES ESTATALES CHILENAS
}

\author{
WOMEN IN DECISION SPACES. CURRENT SCENARIO IN CHILEAN STATE \\ UNIVERSITIES
}

\author{
Susana Riquelme Parra*
}

\begin{abstract}
Resumen
Este estudio aborda la participación de mujeres en espacios de decisión en las Universidades estatales chilenas las cuales forman parte de la administración del Estado. Estas instituciones tienen múltiples funciones orientadas en construir y compartir conocimientos, contribuyendo en el fortalecimiento de la democracia y el desarrollo de la sociedad. No obstante, como instituciones no están exentas de dinámicas estereotipadas en los ámbitos disciplinares y organizacionales, dejando en una posición de subrepresentación a las mujeres en espacios de toma de decisiones, por lo que las funciones desarrolladas por las Universidades se efectúan desde un solo punto de vista.

Por lo anterior, se desarrolla un recorrido por la historia reciente de las Universidades, marcadas por la movilización feminista del año 2018, así como un marco teóricoreferencial que permite la comprensión de las dinámicas de subordinación de las mujeres en las Universidades. Se analiza de manera descriptiva la presencia de mujeres en rectorías, juntas directivas/consejos superiores, decanaturas y autoridades superiores, cuantificando y clasificando su participación en dichos espacios de decisión, según las proporciones y tensiones organizacionales políticas, académicas y administrativas.
\end{abstract}

Palabras clave: Mujeres, Género, Universidades estatales, Espacios de decisiones, Educación Superior.

\begin{abstract}
This study addresses the participation of women in decision-making spaces in Chilean State Universities, which are part of the State administration. These institutions have multiple functions aimed at building and sharing knowledge, contributing to the strengthening of democracy and the development of society. However, as institutions they are not exempt from stereotyped dynamics in the disciplinary and organizational spheres, leaving women in a position of underrepresentation in decision-making spaces, so that the functions developed by the Universities are carried out from a single point of view. view.

Therefore, a journey through the recent history of the Universities is developed, marked by the feminist mobilization of 2018, as well as a theoretical-referential framework that allows the understanding of the dynamics of subordination of women in the Universities. The presence of women in rectories, boards of directors / superior councils, deanships and superior authorities is descriptively analyzed, quantifying and classifying their participation in said decision-making spaces, according to the proportions and political, academic and administrative organizational tensions.
\end{abstract}

Keywords: Women, Gender, State Universities, Decision spaces, Higher Education.

Recibido: 23 de octubre de 2021 / Aceptado: 12 de enero de 2022

\footnotetext{
* Administradora Pública, mención en Ciencia Política, Magíster en Política y Gobierno, Doctoranda en Política y Gestión de la Educación Superior de la Universidad Nacional Tres de Febrero, Argentina. Integrante de Red de Politólogas Chile. Correo srparra@ubiobio.cl. Código ORCID: https://orcid.org/0000-0003-0730-2987
} 


\section{Introducción}

\subsection{Problematización. Las Universidades y las mujeres, escenario actual.}

Esta investigación se basa en el estudio de la participación de las mujeres en espacios de decisión en las Universidades estatales chilenas, entendiendo estos como espacios donde se intersectan las tensiones políticas, académicas y administrativas, por tanto, se abarcan relaciones de poder en función de las lógicas disciplinares y de la organización universitaria.

Las universidades son instituciones complejas de gobernar puesto que cumplen funciones de investigación, docencia creación artística, innovación, extensión con el medio y territorio, lo que requiere ser desarrollado con énfasis en la calidad de su quehacer y se llevan a cabo mediante procesos burocráticos que permiten mantener en pie todas las funciones. Se estudia la situación de las Universidades del Estado, dado su carácter estatal, las cuales tienen un marco jurídico especial a través de la Ley 21.094, la que define explícitamente que son instituciones que forman parte de la administración del Estado.

Los movimientos estudiantiles han dejado de manifiesto las diferentes demandas históricas acerca de las desigualdades, la mercantilización, el lucro y la calidad de ésta en Chile, no obstante, la movilización universitaria del año 2018 en Chile tiene un prisma diferente, puesto que dio visibilidad a otra gran problemática, y corresponde al sexismo en la educación superior.

La movilización estalla a partir de la denuncia de estudiantes de la Universidad Austral de Chile hacia profesores a quienes acusan de acoso sexual. Esta denuncia no surge de manera espontánea, ya que las advertencias comienzan de manera diferenciada en otras Universidades, tales como la Universidad de Chile en el año 2015 a través de "funas" y acciones contra profesores universitarios (Hinner \& López Dietz, 2021; p.118), sin embargo, la movilización del año 2018 logra tal alcance que incide en masivas protestas, "tomas" de edificios, y diferentes acciones de protestas a lo largo de todo el país.

Además de lograr la adhesión de académicas y funcionarias de las comunidades universitarias, las demandas del movimiento feminista se materializan en las Universidades a través del impulso de una institucionalidad que contribuya en la disminución de las desigualdades y en propender a la generación de espacios no sexistas. Las instituciones que no contaban con Direcciones de Género comienzan procesos de instalación de éstas, a cargo de especialistas en el área. El Consejo de Rectores de las Universidades Chilenas (CRUCH) toma como acuerdo la generación de una Comisión dedicada a ello y que promueve el desarrollo de políticas de equidad de género. Junto a ello, se observan acuerdos comunes en las Universidades, como la incorporación (o mejora) de protocolos contra la violencia de género y acoso sexual; implementación de cursos de género, sensibilización y/o capacitación; disminución de contratos de profesores con demandas de acoso; respeto por el nombre social, y, apoyo para estudiantes pares y madres (De Fina \& Figueroa, 2019).

También se logra la tramitación en el Congreso Nacional de la Ley sobre Acoso Sexual, Violencia y Discriminación de Género promovida por académicas de la Red de Investigadoras, ley que se promulga en septiembre de 2021 y que busca la creación de políticas integrales de género y el desarrollo de mecanismos de prevención y sanción, así como de reparación de las víctimas. 
Estas acciones, si bien no abarcan todas las expresiones de desigualdad en las Universidades y tampoco consideran todas las demandas del movimiento feminista, son avances que contribuyen en el abordaje de problemáticas relacionadas a la segregación, violencia y discriminación sexista manifestadas en la educación superior, que corresponden a aquellas que son producto de relaciones de poder, convivencia y construcción de conocimientos que responden a lógicas androcéntricas estructurales, por tanto requieren un abordaje sistémico y complejo de largo alcance, tanto en la problemática más visible de la violencia como de aquellas subordinaciones y exclusiones históricas, así como los dilemas, debates y tensiones de la educación superior en los puestos de toma de decisiones, donde las mujeres se encuentran subrepresentadas, y, de acuerdo con Saracostti (2006), se debe a las tensiones entre trabajo y familia, o "enfrentamientos" entre lo público y lo privado, que limitan y ponen barreras en la carrera por el poder de las mujeres.

\subsection{Consideraciones teórico-referenciales}

Los puntos de vista que ayudan a comprender la problemática acerca de las relaciones de poder organizacional y disciplinar que excluye a las mujeres de los espacios de decisión universitario, son variados. Uno es desde el contrato sexual en los términos de Carole Pateman quien lo describe como una historia de sujeción a partir del pacto patriarcal de los varones sobre las mujeres (Pateman, 1988) y que, en la academia incide en un espacio androcentrista en el cual se reproducen prejuicios y estereotipos de género donde se pone de manifiesto una importante exclusión hacia las mujeres, justificada desde la concepción de la ciencia, dando lugar a dicotomías basadas en la diferencia sexual y un sistema jerarquizado de género. De acuerdo con Diana Maffía los pares dicotómicos dejan fuera de lugar a las mujeres y cuerpos feminizados y no hegemónicos dado que se ubican en lo subjetivo, emocional, privado, metafórico, etc., lejos de las características masculinas como la objetividad, la racionalidad, lo universal, entre otros, que caracterizan lo público y la ciencia (Maffía, 2008).

De la mano de lo anterior, la gobernanza universitaria y la consideración de mujeres como pares y sujetas políticas y científicas, se torna complejo en una institución en la cual subyace la exclusión, y que, si bien con los procesos actuales de masificación de la matrícula universitaria se ha tendido a feminizar la matrícula, esto implica observar si esta transformación (que se encuentra en contexto de pregrado) ha logrado permear la organización universitaria administrativa a través de la participación de mujeres en los espacios de decisión una vez que son parte de la población académica.

\subsubsection{Las mujeres y su posición en la academia. Entre el conocimiento y el poder}

La sustancia básica de la Universidad es el conocimiento, por tanto, comprender la organización de esta institución requiere tener en cuenta las implicancias de su organización, es por ello, que tradicionalmente se organizan a través de Facultades, luego a través de Unidades Académicas. Lo cierto es que organizar el ámbito académico considera romper con las lógicas organizacionales tradicionales ya que son instituciones complejas. 
Analizar las violencias y exclusiones simbólicas de carácter androcéntrico y que forman parte estructural de la universidad como institución, implica situarse en un escenario complejo de abordar, dado que las asimetrías de poder se dan por aspectos disciplinares y por las implicancias de la gestión universitaria.

El conocimiento y las disciplinas en sí mismas son diversas, pero, de acuerdo con el pensamiento desarrollado por la epistemología feminista, el conocimiento situado y la ciencia, no han sido neutrales ni objetivos como pareciera; responden a momentos históricos, contextos materiales y culturales, y, por tanto, situaciones de dominación vinculándose en la relación entre el conocimiento y el poder, en la cual la representación de la realidad es androcéntrica (Suárez Tomé, 2016). Por tanto, las racionalidades que definen los ámbitos organizacionales, atravesados por el conocimiento y su organización disciplinar definidos a través de un pensamiento parcializado y estereotipado, se desarrollan históricamente sin las mujeres, dada su tardía inserción en la producción del conocimiento y porque no ha sido considerada sujeto epistémico (Maffía \& Suárez Tomé, 2021).

\subsubsection{Gobernanza universitaria: Disputas y tensiones con las "intrusas"}

El concepto "intrusa", refiere a una "persona de sexo femenino que se entromete e interviene de forma indeseada en un espacio" (Belausteguigoitia, 2013), y corresponde al título de una de las obras de Ana Buquet (2013), quien aborda las relaciones de género en las universidades desde diferentes puntos de vista y separado por "ámbito" o por población, visualizando interferencias, pausas y retrasos en las carreras académicas, condiciones de trabajo desiguales, segregación y hostigamiento, pero sobretodo, baja representación de las mujeres en tanto cantidad (especialmente en la academia) así como en cargos de toma de decisiones, premios, o espacios simbólicos que manifiestan prestigio y reconocimiento.

Las "intrusas" en la academia, se encuentran en una situación de subordinación histórica. El feminismo se ha abocado a develar esta situación en todos los ámbitos, estructuras e instituciones sociales (Facio, 1999), el androcentrismo cultural y del lenguaje da sustento a la acción simbólica de subordinación (Lamas, 1998), pero las acciones de carácter sexista y de dominación social de los hombres en la vida privada y pública ha determinado una visión sesgada tanto en el quehacer como en la construcción de conocimientos; en este último punto, la academia históricamente se ha constituido como un lugar donde se abordan y debaten las verdades, y donde se desarrolla el conocimiento, pero se ha construido desde una visión parcializada, impidiendo el punto de vista de las mujeres, condicionando las visiones de mundo, los constructos teóricos, epistémicos, ontológicos, de visiones de la realidad e interpretación de las experiencias de vida para dar explicación a los fenómenos sociopolíticos, entre otros.

\subsubsection{Visibilizar las circunstancias y concientizar sobre las exclusiones. Aportes desde el feminismo}

El feminismo se ha preocupado de criticar la naturalización de las posiciones de subordinación de las mujeres. Carole Pateman (1988) aborda la cuestión del contrato social-sexual, que se analiza en este estudio de la mano de la construcción del conocimiento, es por ello que es tan importante la crítica que desde allí se realiza, con obras como las de Sandra Harding (1997) que define al feminismo como un movimiento 
político para el cambio social, y aborda la cuestión de las paradojas de la ciencia. Donna Haraway (1991) define esta labor e identidad del feminismo como difícil de calificar, dado que las subordinaciones son múltiples y se han construido desde discursos científico-sexuales para montar las bases patriarcales, colonialistas y capitalistas, es por ello que no hay una sola matriz de dominación, sino que varias y fragmentadas, donde se encuentran distintos cuerpos, profesionales, de clase, femeninos, radicales, de mediana edad, de distintas localidades, lo que explica los diferentes momentos y aportes que el feminismo realiza respecto a la sexualización de la ciencia, la política y los diferentes espacios en los cuales las mujeres "no encajan".

Diana Maffía (2007) explica algunos momentos en los cuales el feminismo ha ido incidiendo no solo en el cuestionamiento del orden patriarcal, sino que, apuntado a transformar la situación de dominación, por ejemplo, el feminismo de los años ' 70 "de la igualdad" apunta a llegar a los espacios en los cuales las mujeres no habían podido llegar, donde se encuentra: el trabajo, el ingreso a la universidad, igualdad educativa, leyes de divorcio, patria potestad, etc., intentando llegar en igualdad de condiciones al mundo público, para lo cual se considera la idea de igualdad formal, la que de acuerdo con Pautassi (2011), tuvo avances pero se mantiene en espera ya que no es suficiente para generar acciones de igualdad material o sustantiva.

En este sentido, el ingreso de las mujeres a la Universidad considera la no existencia de barreras formales, sin embargo, la pregunta que se efectúa Maffía (2007) es si es que realmente estamos ante iguales condiciones de ingreso, acaso " ¿no existe cierta falacia en esta igualdad?". Es por este (y otros motivos) que en los años '80 el feminismo de la diferencia presenta algunas ideas que abordan la cuestión compleja de la dominación de las mujeres: no existe igualdad, las mujeres no son todas iguales, tampoco se requiere se trate a todas como iguales ante la diferencia de exclusiones, intenta deshegemonizar "lo femenino" y "la mujer", lo cual, en los años '90 el feminismo crítico discute no solo la idea dicotómica-sexualizada (hombre-mujer, masculino-femenino) del orden social, sino que se remite a las múltiples y complejas interacciones que requieren "soltar" los estereotipos con los cuales se han construido realidades y el poder social.

Maffía \& Suárez Tomé (2021) desglosan lo anterior en: contexto social dado, donde se encuentran las instituciones desde el punto de vista estructural; coordinación práctica social, donde la estructura social se encuentra coordinada, y, en la existencia de un ejercicio de control sobre un objeto coordinado, que, implican el imaginario social desde el punto de vista del poder identitario de género, por tanto existe un control sobre las acciones de la mujer basado en estereotipos y prejuicios de género basados en la experiencia social que implica prejuicios que calan lo estructural, por tanto, plantean una opresión sistémica planteada por las identidades, expresiones y roles de género desde el momento de nacer.

Las autoras plantean la importancia de desenmascarar el androcentrismo de la ciencia, lo cual, también permite comprender el entramado que subyace las relaciones de poder en la academia, aludiendo a cinco puntos centrales: (i) los sujetos productores de conocimiento "hombre, europeo o norteamericano, adulto, blanco, cisexual, heterosexual y propietario"; (ii) valor de la racionalidad y emocionalidad, aludiendo a que lo confiable es la razón (hombre) y lo cuestionable y subjetivo es la emoción (mujer); (iii) relación de los cuerpos con el conocimiento, aludiendo a la exclusión de las mujeres por sus características biológicas y "emocionales" asociadas a: menstruación, embarazo, parto, lactancia, orgasmos femeninos y aborto, como aspectos observados por hombres y generadores de exclusión del espacio público; (iv) impacto en la diferenciación de la 
producción y aplicación del conocimiento científico-tecnológico entre hombres y mujeres, dadas las anteriores categorías estereotipadas, y (v) apropiación social de los saberes de acuerdo al género.

\subsubsection{Complejidad, segregación de género y gobernanza universitaria}

Las universidades, se encuentran ante el desafío constante de la excelencia, de hacer frente a los escenarios sociopolíticos, culturales y económicos, de investigar, vincularse con el medio y formar con la responsabilidad de comprender los problemas polifacéticos de las sociedades. De acuerdo con (Lemaitre del Campo \& Durán del Fierro, 2013), en Chile se reconocen tres propósitos fundamentales: (i) la generación de conocimiento, (ii) difusión del conocimiento, y, (iii) desarrollo social y económico.

En este marco, se sitúa al conocimiento como la principal "sustancia" de la educación superior y las universidades. Burton Clarck (1991) define cuatro características acerca del conocimiento y su complejidad. La primera, es su carácter especializado, ya que a medida que avanza el conocimiento, este va conduciendo hacia su división ilimitada. La segunda característica es la autonomía respecto a los campos del conocimiento, con puntos de articulación y otros de distanciamiento; en tercer lugar, plantea lo abierto e incierto que implica el conocimiento como base del descubrimiento, para lo cual el profesorado goza del privilegio de controlar a su manera el tiempo para investigar, y, una cuarta característica es la transmisión del conocimiento como herencia ancestral y cultural, es decir, el conocimiento tiene raíces históricas que se transmite por muchas generaciones.

Las características planteadas por Clarck (1991) ponen de manifiesto la complejidad de la organización académica y sus configuraciones jerárquicas. Este punto de partida es fundamental para comprender que, además de complejas, se enmarca en relaciones dinámicas y laxas, dado que estamos ante una estructura organizacional que intenta "ordenar" lo que en esencia requiere autonomía para el óptimo desarrollo del conocimiento, y donde, además, se está en presencia de "territorios académicos" (Becher, 2001) con sus propias identidades y sistemas de valores. Todas estas particularidades, coexisten en una Universidad, cuya estructura es débilmente acoplada (Weick, 2009).

Para abordar este sistema complejo de organización, se han desarrollado reflexiones acerca de la idea de "gobernanza universitaria", concepto que puede tener diferentes interpretaciones puesto que se encuentra en una etapa de construcción (GangaContreras \& Nuñez-Mascayano, 2018). Lo cierto es que, desde la ciencia política, gobernanza está relacionada directamente con el concepto de gobernabilidad, pero en un sentido complementario, no como sinónimos. Por una parte, gobernabilidad alude a la habilidad de un sistema de gobernarse a sí mismo, y gobernanza, al proceso, las interacciones y las "reglas del juego"; todo esto en contexto de actoras y actores sociales, esperando entonces, cierto equilibrio en la aplicación y evaluación de estas acepciones (Launay-Gama, 2016).

Por tanto, la gobernanza no es un parámetro para medir, sino que se utiliza desde un sentido analítico que contribuye en el entendimiento de los procesos, funciones y contextos, más que de la imposición de un modelo (Launay-Gama, 2016). Esto es relevante ya que, en 1983, Burton Clark ya advierte que las instituciones de educación superior "son de base pesada y con un laxo tejido institucional, donde los consensos son débiles y los compromisos de los actores livianos. El poder está muy diluido y el liderazgo del rector o de la rectora es muy acotado" (Clark, 1983). 
En este sentido, las mujeres se encuentran en una posición de desventaja en el mundo académico, dada la segregación vertical, horizontal y "techos de cristal" que se genera en las comunidades universitarias. De acuerdo con Gorgoso, et. al (2020), la segregación vertical se explica en la medida que avanza la carrera académica, donde va disminuyendo la participación de mujeres mientras los grados y niveles de jerarquización avanzan, concentrandose los hombres en los máximos niveles y también en los puestos de toma decisiones; la segregación horizontal corresponde a la separación estereotipada de mujeres y hombres de acuerdo con los tipos de ciencias, donde las mujeres se concentran más en aquellas "blandas" y los hombres en las "duras"; y, los "techos de cristal" vienen a ser una metáfora que alude a las barreras invisibles que evitan el liderazgo de las mujeres en la academia, limitando su progreso en terminos académicos y en puestos de decisión, debido a prejuicios y discrminaciones, falta de redes de apoyo, estabilidad laboral, etc. (Quiroga-López et. al, 2020).

De acuerdo con el análisis de (Virgili Lillo, Ganga Contreras, \& Figueroa Aillañir, 2015), gobernanza es un concepto multívoco, que se ha entendido como la forma en cómo se ejerce el poder del gobierno y que determina la toma de decisiones de este. En contexto universitario, se plantea la idea de cogobierno universitario, donde las autoras señalan el "biestamental" y el "triestamental", el primero compuesto por académicas/os y estudiantes, y el segundo por académicas/os, estudiantes y funcionarias/os administrativas/os; este último daría cuenta de la articulación de la comunidad universitaria para la toma de decisiones en un marco de democracia o democratización de los espacios universitarios. En estos espacios biestamentales o triestamentales, se visualiza lo que Eagly \& Carli (2004) define como desproporción entre hombres y mujeres explicada, para el caso de la academia, por los techos de cristal y también por los "laberintos de cristal" que alude a los obstáculos que deben sortear las mujeres cuando llegan a espacios de poder como los gobiernos universitarios.

Para Obeide (2020), las complejidades de la gobernanza se reflejan en las relaciones que se dan especialmente entre académicas/os y administrativas/os, para lo cual existen tres ámbitos de trabajo con diferentes racionalidades: el ámbito académico, ámbito administrativo y ámbito político.

Estos ámbitos se caracterizan por su quehacer al interior de la Universidad, el rol y forma de trabajar en cada uno. Para el caso del ámbito académico, este se desataca por la autonomía que, a diferencia del ámbito administrativo, le permite organizar su tiempo para las diferentes actividades académicas que desarrolla, a fin de poder generar conocimiento de frontera así como nuevos análisis e interpretaciones de los fenómenos sociales; para el ámbito administrativo, esta dinámica se distancia de su estructurada forma organizacional, más jerárquica y sin tanta autonomía, dado que cumplen principalmente procedimientos con rutinas más claras, lo que genera una tensión entre ambos ámbitos.

Para el caso del ámbito político, este se encuentra compuesto por autoridades unipersonales y colegiadas. En este ámbito se visualizan aquellas autoridades elegidas por votación, como en el caso de las/os rectores, pero tiene como característica su difuso poder y representación de intereses; en este grupo también se encuentran un "poder informal" que es representado por diferentes actoras/es universitarios, desde representantes gremiales, lealtades personales, sectoriales, incluso partidistas. 


\subsection{Objetivo}

Esta investigación tiene como objetivo cuantificar la participación de las mujeres en puestos de decisión de las Universidades del Consorcio de Universidades Estatales de Chile (CUECH) en el periodo posterior del movimiento feminista del año 2018.

Para ello se desarrolla abarcando el periodo ex post del movimiento feminista del año 2018, examinando la presencia y/o ausencia de mujeres en los puestos de poder y de toma de decisiones institucionales, a fin de tener una mirada sobre la transformación efectiva de estos espacios y acerca de la transversalización de las demandas feministas en la estructura universitaria.

\section{Método}

Se desarrolla un análisis descriptivo, dado que se utiliza información que buscan resumir información de manera tal que refleje de manera objetiva (Rojo, 2006) la participación de mujeres en puestos de decisión en las Universidades del CUECH, para lo cual se considera el uso de fuentes secundarias de información, donde la variable independiente es la movilización feminista de 2018, y la variable dependiente corresponde a las Universidades del CUECH, siendo las mujeres la unidad de observación.

Para ello se efectúa la recopilación de datos de la totalidad de las Universidades del CUECH en cuatro de cargos de poder: rectorías, juntas directivas, decanaturas y autoridades superiores. Este recorte, se relaciona con el propósito de visibilizar posibles brechas y/o avances, esperando contribuir en adelante en el desarrollo de estudios que profundicen en las razones de las composiciones de estos espacios de poder.

Quivy (2005) señala esta decisión metodológica posibilita el análisis de un fenómeno a nivel macro. Los recopilados a través de los repositorios digitales institucionales que contienen información acerca de las autoridades universitarias, son medibles cuantitativamente, sin embargo, tienen como limitante que no explica en términos cualitativos las dinámicas de poder, sino que cuantifica la revisión de documentos y antecedentes dispuestos por las Universidades a través de sus páginas webs.

Para la interpretación de dichos datos, se clasifica y analiza de acuerdo con el planteamiento de Obeide (2020), y de Buquet, Mingo, Cooper y Rodríguez (2010). Obeide permite detectar las tensiones en los ámbitos de poder y racionalidades en el marco de las universidades, los cuales dependen del quehacer y rol institucional de los grupos de personas que trabajan en las Universidades, que dicen relación con esquemas culturales y jerárquicos; por otro lado Buquet, Mingo, Cooper y Rodríguez apuntan a la participación de mujeres en espacios de decisión, los cuales serán clasificados para que, a través de la cuantificación de esto, sea posible señalar si se está ante una institución académica feminizada o masculinizada.

De acuerdo con Andauiza, Crespo y Méndez (2009), la estrategia de análisis es de carácter extensivo ya que solo se observan algunas propiedades, las que en este caso corresponde a la participación de mujeres en espacios de decisión.

El total de casos corresponde a 18 Universidades. Para el levantamiento de los datos, se indaga en las nóminas oficiales de las Universidades del CUECH en el periodo ex ante (solo en el caso de rectorías) y ex post del movimiento feminista del año 2018, de 
acuerdo con la información de sus páginas webs oficiales, con fecha de corte de información: 01 de octubre de 2021.

Se define estudiar a las Universidades del Estado, dado su carácter estatal, las cuales se tienen un marco jurídico especial a través de la Ley 21.346, la que define explícitamente que son instituciones que forman parte de la administración del Estado y que se relacionan con el Presidente de la República a través del Ministerio de Educación.

\section{Resultados}

Al profundizar en el análisis de Obeide (2020) de manera complementaria con autores como Weick (1976), Clark (1991), Becher (2001), de acuerdo con lo que Mónica Marquina (2021) define dentro de los ámbitos de la organización universitaria, se pueden detectar tensiones cruzadas por ámbitos y procesos. En este punto, surgen las posiciones de poder y posibilidades de liderazgo donde indaga la participación de las mujeres.

Cuadro 1: Disputas y tensiones en las Universidades

\begin{tabular}{|l|l|l|l|}
\hline $\begin{array}{l}\mathrm{N}^{\circ} \text { de } \\
\text { Tensiones }\end{array}$ & Ámbito & Ámbito & Definición de la tensión \\
\hline 1 & Político $\Rightarrow$ & Académico & $\begin{array}{l}\text { Elección de autoridades } \\
\text { unipersonales y colegiadas a } \\
\text { través del voto, lo que } \\
\text { conlleva a una confusa y } \\
\text { dispersa idea de autoridad- } \\
\text { par, competitividad y } \\
\text { difusas líneas de jerarquía. }\end{array}$ \\
\hline 2 & Académico $\Rightarrow$ & Político & $\begin{array}{l}\text { Las lealtades están } \\
\text { principalmente con sus } \\
\text { disciplinas académicas, } \\
\text { valorando las evaluaciones } \\
\text { de pares; son autónomos, } \\
\text { por tanto hay una compleja } \\
\text { relación en términos de } \\
\text { planificación, evaluación y } \\
\text { organización de su trabajo } \\
\text { requerido por las } \\
\text { autoridades. }\end{array}$ \\
\hline 3 & Político $\Rightarrow$ & $\begin{array}{l}\text { Administrativo } \\
\text { anterés por llevar a cabo los } \\
\text { programas de gobiernos } \\
\text { universitarios y/o } \\
\text { compromisos de gestión, los } \\
\text { cuales se ven "trabados" por } \\
\text { procedimientos burocráticos } \\
\text { de carácter administrativo, y } \\
\text { por "poca comprensión" del } \\
\text { quehacer de la academia. }\end{array}$ \\
\hline
\end{tabular}




\begin{tabular}{|l|l|l|l|}
\hline 4 & Administrativo $\Rightarrow$ & Político & $\begin{array}{l}\text { Se considera que las } \\
\text { autoridades políticas } \\
\text { desconocen los } \\
\text { procedimientos de } \\
\text { planificación y gestión, } \\
\text { requerimientos de entidades } \\
\text { gubernamentales, y todo el } \\
\text { encadenamiento } \\
\text { administrativo que } \\
\text { conllevan la } \\
\text { implementación de las } \\
\text { acciones (presupuestos, } \\
\text { tiempos, legislación, etc.) }\end{array}$ \\
\hline 5 & Académico $\Rightarrow$ & Administrativo & $\begin{array}{l}\text { Se mueve de manera } \\
\text { autónoma en su quehacer, } \\
\text { por lo que la tensión se } \\
\text { presenta al ser interpelado } \\
\text { bajo mecanismos de control } \\
\text { y planificación en la lógica } \\
\text { de una estructura jerárquica } \\
\text { y rutinizada. }\end{array}$ \\
\hline 6 & Administrativo $\Rightarrow$ & Académico \\
& & $\begin{array}{l}\text { Al encontrarse organizado } \\
\text { en un modelo burocrático } \\
\text { clásico, rutinario y con una } \\
\text { estructura jerárquica } \\
\text { piramidal-formal, colisiona } \\
\text { con el modelo laxo } \\
\text { académico, generándose } \\
\text { tensiones por las diferencias } \\
\text { en las rutinas laborales. }\end{array}$ \\
\hline
\end{tabular}

Fuente: Elaboración propia en base a Obeide (2020) y análisis de Marquina (2021)

Al observar los resultados acerca de la participación de las mujeres en puestos de decisión en las Universidades estatales, se visualiza desde el punto de vista de las proporciones que muestran la composición de estos espacios, que, pese a la alta incidencia del movimiento feminista del año 2018 como impulsor del desarrollo de políticas que contribuyan a la equidad de género y a prevenir, sancionar y erradicar el acoso sexual, violencia y discriminación de género (Ley 21.369), la transformación de los espacios de poder se encuentra todavía pendiente, ya que, según las proporciones que definen los espacios académicos en términos de equilibrio de género (entre el $40 \%$ y 60\%) las Universidades estatales son altamente masculinas.

De acuerdo con Buquet, Mingo, Cooper y Rodríguez (2010), cuando un sexo supera el $60 \%$ en un espacio, se encuentra sobrerrepresentado, mientras que, el sexo cuya participación se encuentre bajo el 40\%, se encuentra subrepresentado.

La elección de rectoras/es, al ser mediante votos, conlleva aquella idea de la primera tensión que se relaciona con simbolismos de autoridad-par, de académicas/os competitivas/os para ganar este cargo y difusas líneas de jerarquía mientras se mantiene en éste. De allí que es relevante el resultado que muestra un cambio favorable (aunque 
aún marginal) a las mujeres como rectoras. En este sentido también es relevante considerar las complejidades de la segregación vertical, lo cual también contribuye a comprender este importante desequilibrio de poder.

Gráfico 1: Rectoras y rectores de Universidades del CUECH

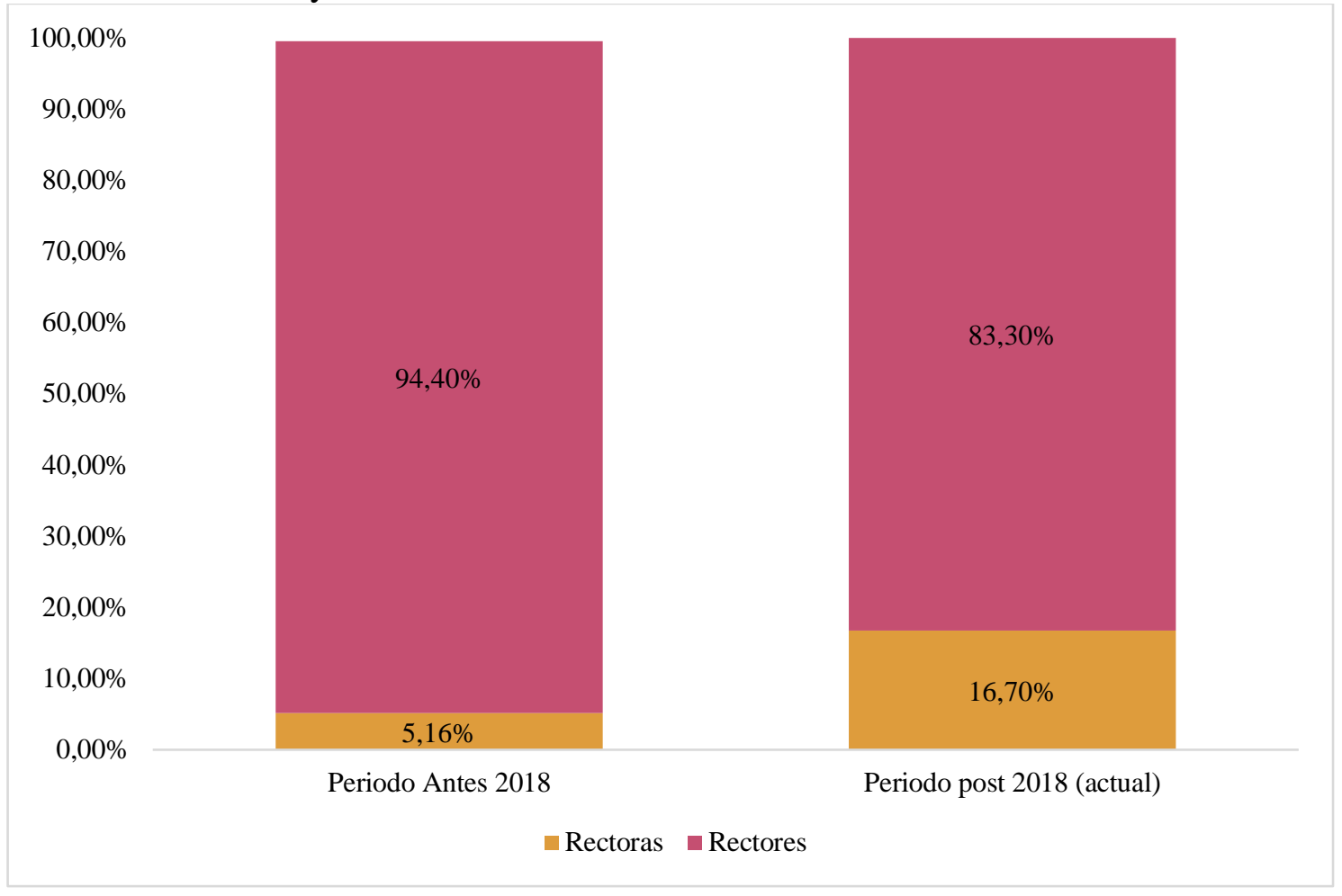

Fuente: Elaboración propia con información de páginas webs de Universidades del CUECH, octubre 2021.

El cargo de rector/a, es uno de los que permite visualizar la tensión 1 y 4 descrita en el Cuadro 1. De acuerdo con el gráfico 1, se observa que, durante el periodo anterior al movimiento feminista del año 2018, en las 18 Universidades del CUECH había solo una mujer rectora, correspondiendo a la Universidad de Aysén, sin embargo, su cargo fue designado por la ex presidenta Michelle Bachelet dado que esta fue creada en el año 2015, luego de la promulgación de la Ley No 20842.

Dado lo anterior, se observa que en el periodo post 2018 son elegidas por el cuerpo académico (de acuerdo a los estatutos de cada institución) las primeras tres rectoras de Chile, correspondiendo a la Universidad Metropolitana de Ciencias de la Educación, Universidad Tecnológica Metropolitana y Universidad de Aysén. Estos resultados son históricos, considerando que ninguna Universidad estatal había sido liderada por una mujer, electa de acuerdo a mecanismos de participación académica, por tanto, son las primeras mujeres que rompen con el orden de acceso al poder en la política universitaria, dominada principalmente por hombres.

No obstante, lo anterior, las rectorías de las Universidades del CUECH son un espacio altamente masculinizado dado que en términos de las proporciones de la "franja paritaria", se observa una sobrerrepresentación de varones como rectores. 
Para identificar la participación de las mujeres en espacios de decisión de las Universidades del CUECH, se decide tres tipos espacios: (i) juntas directivas o consejos superiores, (ii) decanaturas y (iii) autoridades superiores. Se opta por estos ya que son aquellos que se visualizan en los niveles más altos de la estructura organizacional.

Gráfico 2: Participación de mujeres y hombres en espacios de decisión de Universidades del CUECH

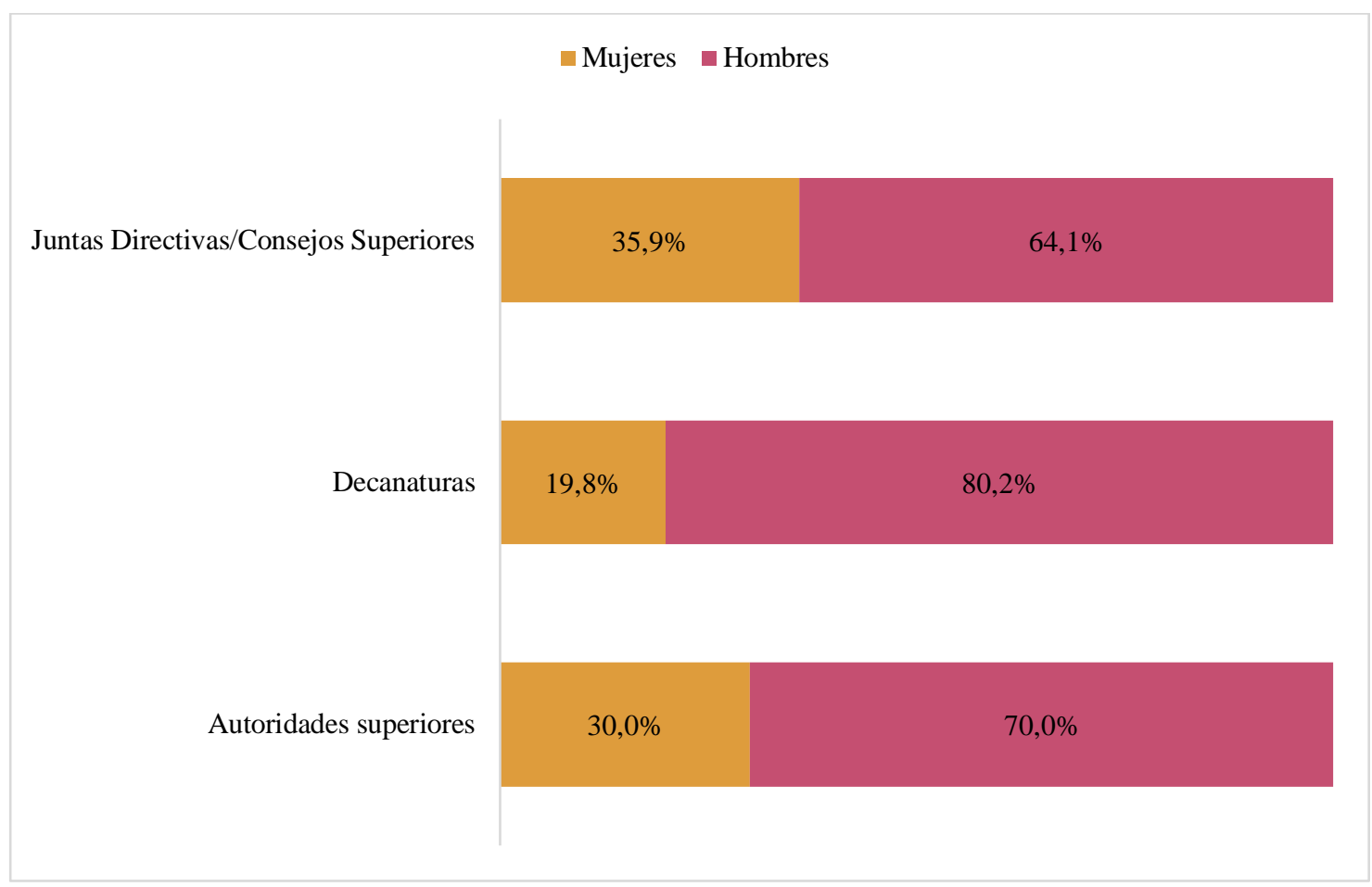

Fuente: Elaboración propia con información de páginas webs de Universidades del CUECH, octubre 2021.

Se visualiza que, las mujeres se encuentran subrepresentadas en los espacios de decisión en las Universidades del CUECH al observar los porcentajes de representación total de estas.

\subsection{Juntas directivas/Consejos superiores}

El principal órgano colegiado de una Universidad estatal corresponde a las Juntas Directivas o Consejos Superiores (de acuerdo a la denominación en sus estatutos universitarios), la diferencia en la nomenclatura, se debe a la vigencia de los estatutos de cada universidad, los cuales, de acuerdo con la Ley de Universidades del Estado deben adecuarse considerando los alcances de esta ley, con obligatoriedad de aquellas que datan desde antes del 11 de marzo de 1990, es decir, que están vigentes desde la dictadura militar. Por tanto, la denominación y composición de los órganos colegiados pueden tener modificaciones luego de junio de 2022 dados los plazos legales para cambiar los estatutos. 
Este órgano colegiado, está compuesto por cuatro tipos de representantes: i) representantes nombrados por el Presidente de la República, ii) integrantes de la comunidad universitaria elegidos mediante sus propios mecanismos, iii) profesional destacado de la región, y iv) el o la rectora.

De acuerdo con la información disponible, se observa que, en las 18 universidades estatales, hay un total de 192 integrantes de las Juntas Directivas o Consejos Superiores. De este grupo, 69 son mujeres y 123 son hombres, lo que indica que el 35,9\% de sus integrantes son mujeres, mostrándose, como un órgano predominantemente masculino. La excepción de esta predominancia se observa en 6 Universidades, de las cuales 2 son paritarias (50\% y 50\%): Universidad de Los Lagos y Universidad de O’Higgins, las otras 4 Universidades se encuentran en los márgenes de la franja paritaria (entre 40\% a 60\%), estas instituciones corresponden a la Universidad de Chile, Universidad del Bío-Bío, Universidad Metropolitana de Ciencias de la Educación y Universidad de Antofagasta, en estos casos, sigue siendo mayor la presencia de hombres. La única excepción se da en la Universidad de Aysén, donde en la composición de este órgano colegiado es predominante la presencia de mujeres $(62,5 \%)$.

\subsection{Decanaturas}

La participación de mujeres como decanas es la más baja luego de las rectorías. Este cargo es unipersonal y elegido por académicas/os integrantes de las diferentes Facultades de las instituciones. En total existen 111 decanaturas en las Universidades del $\mathrm{CUECH}^{2}$, donde 22 están lideradas por mujeres, correspondiendo al 19,8\%. Las decanas, pertenecen a Facultades de humanidades, educación, ciencias sociales, jurídicas, empresariales y ciencias de la salud y odontología, y casos puntuales pertenecen a Ciencias (2 decanas), Forestales (1), Química (1), Farmacia (1), Agrarias (2) e Ingeniería (1).

Al detenerse por institución, se observa que en dos Universidades hay una composición paritaria (50\% y 50\%) de las decanaturas, estas son la Universidad de La Serena y la Universidad Metropolitana de Ciencias de la Educación, y solo una institución muestra una composición, que, estando dentro de la franja paritaria (40\%-60\%) tiene mayor participación de mujeres que de hombres, correspondiendo a la Universidad de Magallanes. El resto tienen proporciones que posicionan a las decanaturas como espacios de representación y decisión académica masculinizadas, visualizándose casos como la Universidad Tecnológica Metropolitana y la Universidad del Bío-Bío donde no hay mujeres decanas.

\subsection{Autoridades superiores}

Se considera como autoridades superiores a rectoras-es, vicerrectoras-es, secretarias-os generales, contraloras-es universitarias-os y otras autoridades que las instituciones describen como autoridades superiores en sus páginas webs oficiales. En este caso, se destaca que existen 120 personas que componen este grupo en las Universidades del CUECH, de quienes 36 son mujeres, correspondiendo al 30\%, lo que indica estar nuevamente en un grupo altamente masculinizado.

\footnotetext{
${ }^{2}$ Las Universidades de O’Higgins, Aysén y Los Lagos, no cuentan con decanaturas, sino que con escuelas y/o carreras que no se encuentran organizadas en Facultades.
} 
La excepción de la predominancia de varones como autoridades superiores en las Universidades del CUECH, corresponde a 4 instituciones: la Universidad de Chile muestra paridad en esta composición (50\% y 50\%), la Universidad Metropolitana de Ciencias de la Educación y Universidad Tecnológica Metropolitana cuya composición se encuentra dentro de la franja paritaria $(40 \%-60 \%)$ donde hay mayor presencia de hombres que mujeres, y la Universidad de Playa Ancha es la única institución cuyas autoridades superiores son principalmente mujeres, representando el $62,5 \%$, siendo entonces, un espacio de decisión donde predominan las mujeres.

Al visualizar el tipo de cargos que tienen las mujeres que son autoridades superiores, se observa que son principalmente Vicerrectoras (20 mujeres), seguidas de Secretarias Generales (8 mujeres), Contraloras Universitarias (2) y Prorrectoras (2). Cabe destacar que en este grupo también se encuentran las-os rectoras-es, correspondiendo a un total de 3, y en el caso de la Universidad de Chile, se presenta como parte de las autoridades superiores a la Vicepresidenta del Senado Universitario.

\subsection{Sintesis: Mujeres en espacios de decisión en Universidades estatales}

Para orientar la clasificación de los espacios de decisión de acuerdo con la participación de las mujeres en cada uno de ellos al interior de una institución universitaria, se sugiere considerar el cuadro 2. Es importante tomar en cuenta que este cuadro aplica para espacios donde se requiere la designación o elección con relación al conjunto de la institución, a fin de tener una mirada de la organización completa.

Cuadro 2: Clasificación de la presencia de mujeres en espacios de decisión

\begin{tabular}{|c|c|c|c|c|l|}
\hline \multirow{2}{*}{$0 \%$} & $1 \%-39 \%$ & $40 \%-49 \%$ & $50 \%$ & $51 \%-60 \%$ & $\begin{array}{l}61 \%- \\
100 \%\end{array}$ \\
\cline { 3 - 5 } & Subrepresentación & $\begin{array}{l}\text { Sepresentación } \\
\text { mujeres } \\
\text { en intervalo } \\
\text { inferior }\end{array}$ & Paridad & $\begin{array}{l}\text { Representación } \\
\text { en intervalo } \\
\text { superior }\end{array}$ & $\begin{array}{l}\text { Espacio } \\
\text { feminizado }\end{array}$ \\
\hline
\end{tabular}

Fuente: Elaboración propia

Cuadro 3: Síntesis de la presencia de mujeres según las tensiones

\begin{tabular}{|l|l|l|l|l|}
\hline $\begin{array}{l}\mathrm{N}^{\circ} \text { de } \\
\text { Tensiones }\end{array}$ & Ámbito & Ámbito & $\begin{array}{l}\text { Espacio de } \\
\text { decisión con } \\
\text { presencia de } \\
\text { mujeres }\end{array}$ & $\begin{array}{l}\text { Clasificación } \\
\text { de la } \\
\text { representación } \\
\text { de las } \\
\text { Universidades } \\
\text { del CUECH }\end{array}$ \\
\hline 1 & $\begin{array}{l}\text { Político } \\
\Rightarrow\end{array}$ & Académico & $\begin{array}{l}\text { Rectorías } \\
\text { Juntas } \\
\text { Directivas }\end{array}$ & \\
\hline 2 & $\begin{array}{l}\text { Académico } \\
\Rightarrow\end{array}$ & Político & $\begin{array}{l}\text { Decanaturas } \\
\text { Autoridades } \\
\text { superiores }\end{array}$ & \\
& & & & 0 \\
0 \\
0
\end{tabular}




\begin{tabular}{|c|c|c|c|}
\hline 3 & $\begin{array}{l}\text { Político } \\
\Rightarrow\end{array}$ & Administrativo & $\begin{array}{l}\text { Autoridades } \\
\text { superiores }\end{array}$ \\
\hline 4 & $\begin{array}{l}\text { Administrativo } \\
\Rightarrow\end{array}$ & Político & $\begin{array}{l}\text { Rectorías } \\
\text { Autoridades } \\
\text { superiores }\end{array}$ \\
\hline 5 & $\begin{array}{l}\text { Académico } \\
\Rightarrow\end{array}$ & Administrativo & Decanaturas \\
\hline 6 & $\begin{array}{l}\text { Administrativo } \\
\Rightarrow\end{array}$ & Académico & $\begin{array}{l}\text { Rectorías } \\
\text { Autoridades } \\
\text { superiores }\end{array}$ \\
\hline
\end{tabular}

Fuente: Elaboración propia

Cuadro 4: Clasificación de las Universidades de acuerdo con la presencia de mujeres espacios de decisión

\begin{tabular}{|l|l|l|l|l|}
\hline Universidad & Rectoras & $\begin{array}{l}\text { Juntas } \\
\text { Directivas/Consejo } \\
\text { s Universitarios }\end{array}$ & Decanaturas & $\begin{array}{l}\text { Autoridades } \\
\text { Superiores }\end{array}$ \\
\hline $\begin{array}{l}\text { Universidad } \\
\text { de Tarapacá }\end{array}$ & $\begin{array}{l}\text { Sin } \\
\text { Mujeres }\end{array}$ & Subrepresentación & Subrepresentación & Subrepresentación \\
\hline $\begin{array}{l}\text { Universidad } \\
\text { Arturo Prat }\end{array}$ & $\begin{array}{l}\text { Sin } \\
\text { Mujeres }\end{array}$ & Subrepresentación & Subrepresentación & Subrepresentación \\
\hline $\begin{array}{l}\text { Universidad } \\
\text { de } \\
\text { Antofagasta }\end{array}$ & $\begin{array}{l}\text { Sin } \\
\text { Mujeres }\end{array}$ & $\begin{array}{l}\text { Intervalo inferior } \\
\text { franja paritaria }\end{array}$ & Sin Mujeres & Subrepresentación \\
\hline $\begin{array}{l}\text { Universidad } \\
\text { de Atacama }\end{array}$ & $\begin{array}{l}\text { Sin } \\
\text { Mujeres }\end{array}$ & Subrepresentación & Subrepresentación & Subrepresentación \\
\hline $\begin{array}{l}\text { Universidad } \\
\text { de La } \\
\text { Serena }\end{array}$ & $\begin{array}{l}\text { Sin } \\
\text { Mujeres }\end{array}$ & Subrepresentación & Paritaria & Subrepresentación \\
\hline $\begin{array}{l}\text { Universidad } \\
\text { de Playa } \\
\text { Ancha }\end{array}$ & $\begin{array}{l}\text { Sin } \\
\text { Mujeres }\end{array}$ & Subrepresentación & Subrepresentación & Espacio feminizado \\
\hline $\begin{array}{l}\text { Universidad } \\
\text { de } \\
\text { Valparaíso }\end{array}$ & $\begin{array}{l}\text { Sin } \\
\text { Mujeres }\end{array}$ & Subrepresentación & Subrepresentación & Subrepresentación \\
\hline
\end{tabular}




\begin{tabular}{|c|c|c|c|c|}
\hline $\begin{array}{l}\text { Universidad } \\
\text { de Santiago } \\
\text { de Chile }\end{array}$ & $\begin{array}{l}\text { Sin } \\
\text { Mujeres }\end{array}$ & Subrepresentación & Subrepresentación & Subrepresentación \\
\hline $\begin{array}{l}\text { Universidad } \\
\text { Tecnológica } \\
\text { Metropolita } \\
\text { na }\end{array}$ & $\begin{array}{l}\text { Con } \\
\text { presencia }\end{array}$ & Subrepresentación & Sin Mujeres & $\begin{array}{l}\text { Intervalo inferior } \\
\text { franja paritaria }\end{array}$ \\
\hline $\begin{array}{l}\text { Universidad } \\
\text { de Chile }\end{array}$ & $\begin{array}{l}\text { Sin } \\
\text { Mujeres }\end{array}$ & Paritaria & Subrepresentación & Paritaria \\
\hline $\begin{array}{l}\text { Universidad } \\
\text { de } \\
\text { O'Higgins }\end{array}$ & $\begin{array}{l}\text { Sin } \\
\text { Mujeres }\end{array}$ & Paritaria & No aplica & Subrepresentación \\
\hline $\begin{array}{l}\text { Universidad } \\
\text { de Talca }\end{array}$ & $\begin{array}{l}\text { Sin } \\
\text { Mujeres }\end{array}$ & Subrepresentación & Subrepresentación & Subrepresentación \\
\hline $\begin{array}{l}\text { Universidad } \\
\text { del Bío-Bío }\end{array}$ & $\begin{array}{l}\text { Sin } \\
\text { Mujeres }\end{array}$ & $\begin{array}{l}\text { Intervalo inferior } \\
\text { franja paritaria }\end{array}$ & Sin Mujeres & Subrepresentación \\
\hline $\begin{array}{l}\text { Universidad } \\
\text { de La } \\
\text { Frontera }\end{array}$ & $\begin{array}{l}\text { Sin } \\
\text { Mujeres }\end{array}$ & Subrepresentación & Subrepresentación & Subrepresentación \\
\hline $\begin{array}{l}\text { Universidad } \\
\text { Metropolita } \\
\text { na de } \\
\text { Ciencias de } \\
\text { la } \\
\text { Educación }\end{array}$ & $\begin{array}{l}\text { Con } \\
\text { presencia }\end{array}$ & $\begin{array}{l}\text { Intervalo inferior } \\
\text { franja paritaria }\end{array}$ & Paritaria & $\begin{array}{l}\text { Intervalo inferior } \\
\text { franja paritaria }\end{array}$ \\
\hline $\begin{array}{l}\text { Universidad } \\
\text { de Los } \\
\text { Lagos }\end{array}$ & $\begin{array}{l}\text { Sin } \\
\text { Mujeres }\end{array}$ & Paritaria & No aplica & Subrepresentación \\
\hline $\begin{array}{l}\text { Universidad } \\
\text { de Aysén }\end{array}$ & $\begin{array}{l}\text { Con } \\
\text { presencia }\end{array}$ & Espacio feminizado & No aplica & Subrepresentación \\
\hline $\begin{array}{l}\text { Universidad } \\
\text { de } \\
\text { Magallanes }\end{array}$ & $\begin{array}{l}\text { Sin } \\
\text { Mujeres }\end{array}$ & Subrepresentación & Espacio feminizado & Subrepresentación \\
\hline
\end{tabular}

Fuente: Elaboración propia 


\section{Discusión}

Las agendas universitarias de Chile y América Latina se encuentran ante el desafío de hacer frente a las nuevas responsabilidades políticas, sociales y académicas que están asumiendo o se asumirán en el futuro, dado que, como instituciones, debieran contribuir con el desarrollo de las sociedades, fortaleciendo la democracia y con justicia social (Fernández Lamarra, 2010).

Una de las claves de los nuevos escenarios es la pertinencia político-institucional de la educación superior en términos de su contribución a la gobernabilidad democrática, que requiere que las instituciones tengan una mejor gobernabilidad interna (Fernández Lamarra, 2021), y dentro ello, cabe preguntarse acerca de las agendas universitarias desde el punto de vista de las mujeres, su posición y participación más allá de su matrícula en pregrado, ya que para que los rasgos dominantes de la sociedad se transformen, se requiere que las brechas y disparidades se erradiquen, de esta manera, la contribución a la sociedad sería con una mirada completa y plural en todos los ámbitos del quehacer de las Universidades y de la educación superior.

\subsection{Tensiones y representación de mujeres en puestos de decisión. Algunas explicaciones}

La participación de las mujeres en puestos de toma decisión que son parte de las Universidades del CUECH es baja. Esto responde a las racionalidades políticas y académicas al interior de las universidades, y a la tardía incorporación de las mujeres en los campos disciplinares visualizándose una doble subordinación y mayores complejidades para ocupar cargos de toma de decisiones.

Esto tiene implicancias en la transformación de las Universidades, ya que ocupar puestos de decisión permite incidir en la comprensión de nuevos contratos originarios de estas instituciones en los términos planteados por Carole Pateman (1988), puesto que el contrato originario sexual-social se visualiza en estas composiciones donde la subordinación de las mujeres es evidente al estar frente instituciones dominadas por hombres.

Esta dominación relacionada con las tensiones políticas-académicas y académicas-políticas, que se traducen en liderazgos, jerarquías, confianzas, validación por parte de pares académicos, etc., tiene orígenes de exclusión e invisibilización de las mujeres como sujeto y objeto de la ciencia, tal como plantea Diana Maffía, plasmándose en las Universidades al ser instituciones que trabajan en torno al conocimiento, y donde ha dominado la visión androcéntrica caracterizada por su racionalidad, capacidad lógica, abstracción, universalización y objetividad, todas estas atribuidas a los hombres, mientras que a las mujeres se alude a todas aquellas características que carecen de valor en términos científicos (y políticos), tales como la subjetividad, sensibilidad, singularidad, narratividad, entre otras (Maffía, 2007).

Para alcanzar una efectiva transformación, se requiere tener en cuenta las lógicas jerárquicas y sus orígenes patriarcales, de esta manera es posible comprender las razones por las cuales las mujeres han estado fuera de la toma de decisiones, así como en diferentes espacios de interacción social (Schuster Ubilla, y otros, 2019) entre los que se encuentra la Universidad.

Dentro de las dimensiones que configuran las demandas del movimiento feminista del año 2018, se encuentran elementos como: educación no sexista, cuidado y trabajo reproductivo, derechos sexuales y reproductivos, violencia sexual y discriminación, y 
subcontrato y condiciones laborales (Miranda Leibe \& Roque López, 2018), y si bien, la respuesta institucional y legal ha ido en post de transversalizar la perspectiva de género a fin de abordar estas demandas, al observar los datos sobre las mujeres en espacios de decisión, se observa que no se ha cambiado ni tocado la estructura de poder de las Universidades, siendo esta una tarea desafiante, puesto que apunta a desestructurar todas las nociones de subordinación estructurales y simbólicas.

Lo anterior implica tener en cuenta las conexiones estructurales entre el desarrollo del conocimiento (segmentado y parcializado), las instituciones, el ejercicio de la ciudadanía y de los derechos, a lo cual subyace una base estereotipada y dicotomizada sobre las características y roles de hombres y mujeres, que repercuten y se replican históricamente sin este cuestionamiento estructural.

Las universidades del Estado son también reflejo del contrato sexual-social en todas sus dimensiones: científicas-disciplinares-políticas-administrativas, etc., puesto que son interacciones que se cruzan con la visión parcializada de la construcción del conocimiento efectuada desde una visión androcéntrica hegemónica de la ciencia y, por tanto, de la construcción de verdades (incluyendo la "verdad" del contrato social) en todas las disciplinas, y esto atraviesa todos los ciclos educativos, reproduciendo sistemáticamente esta mirada estereotipada, contribuyendo a perpetuar el contrato sexual dadas las raíces patriarcales de estos contratos.

Es por ello que, el movimiento feminista del año 2018 ha sido fundamental en empujar las agendas universitarias a transformar las instituciones por unas en las cuales se prevenga y sancionen todas aquellas prácticas de violencia y discriminación de género, sin embargo, el desafío se encuentra en la transformación de los espacios de decisión para que las mujeres puedan permear desde sus miradas los diferentes espacios políticos, académicos y administrativos que hoy se visualizan como tensiones (Obeide, 2020), pero que como tales, son masculinas.

En términos de gobernanza de las universidades estatales en Chile, se considera relevante que contribuyan en el ejercicio de administrar la institución en términos de equilibrio entre lo político y académico, pero a su vez, en términos de la posibilidad de dirigir con capacidad política acorde a los "nuevos" tiempos y escenarios, de manera competente, considerando el diálogo, la discusión, entendimiento, negociaciones y compromisos con los actores, generando empoderamiento, coordinación, colaboración y corresponsabilidad (Aguilar, 2010). "La pertinencia y eficiencia en que se encaren las nuevas responsabilidades políticas, sociales y académicas de la educación favorecerán, o no, sus deseables contribuciones a un pleno desarrollo en democracia y con justicia social de los países de América Latina" (Fernández Lamarra, s/f).

Todo ello, en términos de equilibrio de género, si no, se mantiene una estructura y espacio de decisión basada en los "viejos tiempos" que excluyen a las mujeres. Se requiere un reconocimiento y representación efectiva de las mujeres en los espacios universitarios, a fin de materializar práctica y teórica, ello contribuiría a sociedades más justas, que convivan armónicamente, ejerciendo efectivamente una democracia completa.

La carencia de poder de las mujeres queda plasmada en la ausencia de estas en los espacios de decisión, y si en el marco universitario esta evidencia no es transformada, difícilmente van a cambiar los criterios que enmarcan las relaciones sociales y democráticas, puesto que en las Universidades se construye el conocimiento que se comparte y socializa, y a su vez, son las instituciones reflejo de la sociedad y con responsabilidad de hacer frente a los problemas y desafíos de ésta, aportando al bien común, sobre todo las instituciones estatales, cuya misión es cultivar, generar, desarrollar 
y transmitir el saber superior en las diversas áreas del conocimiento y dominios de la cultura, por medio de la investigación, la creación, la innovación y de las demás funciones de estas instituciones (Ley 21.094).

Como desafío de esta investigación, se encuentra la indagación sobre las relaciones de poder al interior de las universidades, los limites y desafíos de las mujeres en términos de liderazgo, indagando en aspectos de carácter simbólico que complementen los datos reflejados en este artículo, abordando así los desafíos y análisis que se propone desde el feminismo para deconstruir las relaciones de raíz patriarcal en las instituciones así como las valoraciones y dicotomías estereotipadas y sexistas, para que de esta manera, se conozca en profundidad las relaciones de poder simbólica al interior de las universidades.

\section{Conclusiones}

El estudio permite concluir que la participación de las mujeres en espacios de decisión en las Universidades del CUECH es débil dado que se encuentran subrepresentadas en todos los espacios estudiados, los cuales se constituyen como altamente masculinizados, por lo que se requiere de acciones que se enmarquen en resolver las amplias brechas visualizadas, que limitan el liderazgo de las mujeres en los ámbitos políticos, administrativos y académicos.

Las diferentes tensiones que asumen las Universidades como instituciones complejas organizativamente, son abordadas históricamente principalmente por hombres. El estudio permite evidenciar ello, pero también observar algunas instituciones que avanzan en la incorporación de mujeres en puestos de decisión, y ello, si bien no está estudiado desde un punto de vista inferencial-causal, si se observa una relación marcada por la historia reciente de las Universidades en la cual el movimiento feminista tuvo una influencia importante no solo en la generación de diagnósticos y políticas institucionales, sino que también en el desarrollo de la ley 21.369.

En el caso particular de la participación de mujeres en espacios de decisión, pese a que todavía se caracteriza por la subrepresentación, se visualiza un avance importante en tres Universidades estatales, donde, el cuerpo académico elige por primera vez a una mujer como rectora, siendo un precedente histórico sobre un proceso transformativo impulsado por mujeres y que apunta a la igualdad de oportunidades y la representación sustantiva.

Las acciones de las Universidades estatales que se enmarquen en principios de equidad de género, tal como mandata la ley, deben desarrollarse asumiendo el desafío de comprender (para desestructurar) la problemática del sexismo en la academia, dados por estereotipos de género y sistemas no formales de subordinación de las mujeres que inciden en las relaciones e interacciones académicas, políticas y administrativas.

Este tipo de estudios muestra el resultado de una estructura jerárquica androcéntrica que requiere profundas transformaciones, y que desafían a continuar desarrollando investigaciones que profundicen en aspectos relacionales, mediante el uso de metodología cualitativas que den cuenta de las implicancias de las mujeres en cargos de poder y los procesos que transformación o estancamiento al interior de las instituciones que permitan indagar en las causas de las relaciones de poder y su influencia en las funciones que desarrollan las Universidades como la docencia, la investigación, la innovación, la creación artística, y, la vinculación con el medio y territorios, así como en 
su contribución al fortalecimiento de la democracia y el desarrollo social, labores que requieren tener una mirada completa.

Finalmente, los espacios académicos donde no hay mujeres o se encuentran subrepresentadas se caracterizan por ser incompletos, con visión y quehacer parcializado. Ello requiere ser desmontado, y para ello se necesita de más estudios y acciones que visibilicen las brechas y abarquen las complejidades de los procesos y tensiones que se dan en las Universidades, contribuyendo a reparar las marcas de exclusión y el sexismo en la educación superior. 


\section{Referencias}

Aguilar, L. (2010). Gobernanza: El nuevo proceso de gobernar. Fundación Friedrich Naumann para la Libertad.

Andauiza Perea, E., Crespo Martínez, I., \& Méndez Lago, M. (2009). Metodología de la ciencia política. Centro de Investigaciones Sociológicas.

Becher, T. (2001). Tribus y territorios académicos. La indagación intelectual y las culturas de las disciplinas. Gedisa.

Belausteguigoitia, M. (2013). Prólogo. En A. Buquet, J. Cooper, A. Mingo, \& H. Moreno, Intrusas en la Universidad (págs. 9-10). UNAM.

Clarck, B. (1991). El sistema de educación superior. Una visión comparativa de la organización académica. Nueva Imagen.

Clark, B. (1983). El sistema de educación superior. Universidad de California.

De Fina. D. F., \& Figueroa F. (2019). Nuevos “campos de acción política" feminista: Una mirada a las recientes movilizaciones en Chile. Revista Punto Género, (11), 51-72.

Eagly, \& Carli. (2004). Women and Men as Leaders. Sage Publications.

Facio, A. (1999). Feminismo, género y patriarcado. En A. Facio, \& L. Fries, Género y Derecho. LOM Ediciones.

Fernández Lamarra, N. (2010). Hacia una nueva agenda de la educación superior en América Latina. Anuies.

Fernández Lamarra, N. (2021). Seminario: Escenarios y políticas comparadas de educación superior|. Doctorado en Política y Gestión de la Educación Superior.

Fernandez Lamarra, N. (s.f.). La educación en América Latina. Una perspectiva comparada. Simposio: Aproximación a la Educación en Latinoamérica (págs. 126). Consejo Argentino para las relaciones internacionales.

Ganga-Contreras, F., \& Nuñez-Mascayano, O. (2018). Gobernanza de las organizaciones: Acercamiento conceptual a las instituciones de educación superior. Espacios Vol. $39 \mathrm{~N}^{\circ} 17,9$.

Vázquez, A. E. G., Suárez, A. P. G., \& Rosabal, E. M. D. (2020). El desarrollo local universitario. Una mirada desde las brechas de género de la Universidad de Granma. LIBRO DE ACTAS, 313.

Haraway, D. (1991). Manifiesto para cyborgs. Ciencia, tecnología y feminismo socialista a finales del siglo XX. Letras Svdaca.

Harding, S. (1997). Ciencia y feminismo. Morata.

Hinner, H., \& López Dietz, A. (2021). Movimientos feministas y LGBTQ+: de la transición pactada a la revuelta social, 1990-2020. En A. Gálvez, Históricas. Movimientos feministas y de mujeres en Chile, 1850-2020 (págs. 91-128). Lom Ediciones.

Lamas, M. (1998). La violencia del sexismo. En A. S. Vásquez, El mundo de la violencia (págs. 191-198). UNAM, Fondo de Cultura Económica.

Launay-Gama, C. (2016). Gobernanza/Gobernabilidad. En M. Picazo, V. Montero, \& J. Simon, Diccionario de Ciencia Política (págs. 151-155). Sello Editorial. Universidad de Concepción.

Lemaitre del Campo, M. J., \& Durán del Fierro, F. (2013). Hacia una nueva arquitectura del sistema de educación superior: el régimen de lo público. Aequalis. Foro de Educación Superior.

Maffía, D. (2007). Epistemología feminista: la subversión semiótica de las mujeres en la ciencia. Revista venezonala de estudios de la mujer, $\mathrm{n}^{\circ} 12(28), 63-98$. 
Maffía, D. (2008). Contra las dicotomías: feminismo y epistemología crítica. Seminario de epistemología feminista (págs. 1-9). Buenos Aires: Instituto Interdisciplinario de Estudios de Género.

Maffía, D., \& Suárez Tomé, D. (2021). Ciencia y epistemología. Curso online: Ciencia y Feminismo. Red Argenina de género, ciencia y tecnología. Economía femini(s)ta. Tierra Violeta.

Marquina, M. (2021). La organización universitaria. Seminario: Desafíos para la política y gestión de la educación superior. Doctorado en Política y Gestión de la Educación Superior.

Miranda Leibe, L., \& Roque López, B. (2018). El mayo estudiantil feminista de 2018 en la Pontificia Universidad Católica de Chile "La revolución es feminista". Activismos feministas jóvenes, 59.

Obeide, S. (2020). Nuevos roles, nuevas identidades: ¿el nacimiento de un nuevo sector en las universidades públicas argentinas? Integración y Conocimiento, Vol. 9, n¹, 101-112.

Pateman, C. (1988). El contrato sexual. Ménades Trincheras.

Pautassi, L. (2011). La igualdad en espera: el enfoque de género. Lecciones y ensayos, $\mathrm{n}^{\circ}$ 89, 279-298.

Quiroga-López, M. K., Poaquiza-Poaquiza, Á. P., \& Altamirano-Altamirano, S. J. (2020). Techos de cristal en la docencia femenina universitaria. Revista Científica y Arbitrada de Ciencias Sociales y Trabajo Social: Tejedora. ISSN: 2697-3626, 3(6 Ed. esp.), 19-31.

Quivy, Raymond (2005). Manual de investigación en ciencias sociales. Limusa, México. Rojo, J. M. (2006). Análisis descriptivo y exploratorio de datos. Laboratorio de Estadística del Instituto de Economía y Geografía Consejo Superior de Investigaciones Científicas, Madrid.

Saracostti, M. (2006). Mujeres en la alta dirección de educación superior: posibilidades, tensiones y nuevas interrogantes. Calidad en la Educación, (25), 243-259.

Schuster Ubilla, S., Santos Pérez, A., Miranda Leibe, L., Roque López, B., Arce-Riffo, J., \& Medel Vera, E. (2019). Una mirada al movimiento feminista en Chile del año 2018: Hitos, agenda y desafíos. Iberoamerixana, XIX, 72, 223-245.

Suárez Tomé, D. (2016). Ciencia y emociones: ¿responde la exclusión de la emotividad en la investigación científica a un prejuicio androcéntrico? Tábano, $\mathrm{n}^{\circ} 12,71-89$.

Virgili Lillo, M., Ganga Contreras, F., \& Figueroa Aillañir, K. (2015). Gobernanza universitaria o cogobierno: El caso de la Universidad de Concepción de Chile. Última década, 187-216.

Weick, K. (2009). Las organizaciones educativas como sistemas flojamente acoplados. Gestión y Estrategia, Num 36, 93-110. 